\title{
La acreditación de sustentabilidad y su impacto en los márgenes de rentabilidad del sector industrial de la Bolsa Mexicana de Valores
}

\author{
The accreditation of sustainability and its impact on profit margins in the \\ industrial sector of the Mexican Stock Exchange
}

\section{A acreditação de sustentabilidade e seu impacto nas margens de rentabilidade do sector industrial da Bolsa Mexicana de Valores}

\author{
José Antonio Morales-Castro ${ }^{1}$
}

Forma de citar: J. A. Morales, "La acreditación de sustentabilidad y su impacto en los márgenes de rentabilidad del sector industrial de la Bolsa Mexicana de Valores”, Respuestas, vol. 20, no. 1, pp. 16-29, 2015.

Recibido:

24 de Junio 2014

Aceptado:

9 de Octubre 2014

\section{Resumen}

Objetivo: El presente trabajo se centró en probar empíricamente la acreditación en las áreas de manejo y uso de recursos naturales, responsabilidad social y gobierno corporativo de las empresas del sector industrial que se necesita para ingresar al índice sustentable de la Bolsa Mexicana de Valores (BMV), y su efecto en la rentabilidad. Metodología: Se identificó las 30 empresas del sector industrial cotizantes en la BMV, se calcularon 6 ratios de rentabilidad para cada una de ellas, después se formaron dos grupos, las que se acreditaron para ingresar al índice sustentable y las que no se acreditaron y se calculó la diferencia estadística del promedio de los valores de los ratios de rentabilidad entre los dos grupos y la diferencia estadística del promedio de los índices de los ratios de rentabilidad para las empresas acreditadas como sustentables para contrastar los periodos previo y posterior a la fecha de ingreso al índice sustentable. Resultados: Se encontró que las empresas acreditadas como sustentables tienen valores más altos en sus ratios de rentabilidad frente a las que no se acreditaron, aunque solo cuatro de ellos tienen diferencia estadística significativa. Conclusión: Certificarse en las áreas de manejo y uso de recursos naturales, responsabilidad social y gobierno corporativo para ingresar al índice sustentable de las bolsas de valores si se refleja en aumento de márgenes de rentabilidad bruta y de operación en las empresas.

Palabras clave: Acreditación de sustentabilidad, Bolsa Mexicana de Valores, Ratios de Rentabilidad, Sector industrial.
Abstract
Objective: This work focused on empirically test the accreditation in the areas of management and use of natural resources, social responsibility and corporate governance of companies in the industrial sector is needed to enter the sustainable index of the Mexican Stock Exchange (BMV) and its effect on profitability. Methods: The 30 companies in the industrial sector contributors in the BMV was identified, 6 profitability ratios for each calculated, then two groups were formed, which were credited to enter the sustainable rate and not credited and calculated the statistical difference in average values profitability ratios between the two groups and the statistical difference in average rates of profitability ratios for firms accredited as sustainable to compare the periods before 
and after the date of entry into index sustainable. Results: It was found that companies accredited as sustainable have higher values in their profitability ratios compared to those not accredited, but only four of them are statistically significant. Conclusion: Certified in the areas of management and use of natural resources, social responsibility and corporate governance to enter the sustainable rate securities exchanges is reflected in increased gross margins and operating profitability in enterprise.

Keywords: Accreditation of sustainability, Mexican Stock Exchange, Profitability Ratios, Industrial sector.

\section{Resumo}

Objetivo: O presente trabalho se centrou em provar empiricamente a acreditação nas áreas de manejo e uso de recursos naturais, responsabilidade social e governo corporativo das empresas do sector industrial que se necessita para ingressar ao índice sustentável da Bolsa Mexicana de Valores (BMV), e seu efeito na rentabilidade. Metodologia: Identificouse as 30 empresas do sector industrial contribuidores na BMV, se calcularam seis (6) índices de rentabilidade para cada uma delas, depois se formaram dois grupos, as que se acreditaram para ingressar ao índice sustentável e as que não se acreditaram, e se calculou a diferencia estadística da media dos valores dos índices de rentabilidade entre os dois grupos e a diferencia estadística da media dos índices de rentabilidade para as empresas acreditadas como sustentável para contrastar os períodos anterior e posterior a data de ingresso ao índice sustentável. Resultados: Encontrou-se que as empresas acreditadas como sustentáveis tem valores mais altos em seus índices de rentabilidade frente as que não se acreditaram, embora só quatro (4) delas apresentaram diferencia estadística significativa. Conclusão: Certificar-se nas áreas de manejo e uso de recursos naturais, responsabilidade social e governo corporativo para ingressar ao índice sustentável das bolsas de valores, só sim se percebe um aumento das margens de rentabilidade bruta e de operação nas empresas.

Palavras-chave: Acreditação de sustentabilidade, Bolsa Mexicana de Valores, Índices de Rentabilidade, Setor industrial.

\section{Introducción}

Aunque el objetivo fundamental de las empresas desde el punto de vista de los accionistas es crear valor para así obtener mayor rentabilidad. Con el tiempo, la teoría de la administración ha integrado las nuevas corrientes del pensamiento que reconocieron una función social de la empresa que trasciende a sus propios accionistas e incorpora al conjunto de los grupos de interés como destinatarios necesarios e ineludibles de sus acciones y a quienes también se deben conformar [1]. Para atender las demandas de los grupos encargados del medio ambiente.
Las empresas buscan mantenerse funcionando a lo largo del tiempo, para recuperar la inversión en los diversos activos, lo cual caracteriza el concepto de empresa sostenible o sustentable. El desarrollo sostenible consiste además de las cuestiones medioambientales, requiere la integración de los tres pilares del desarrollo: el crecimiento económico, el progreso social y los aspectos medioambientales [2], las compañías que cumplen estos aspectos, tienden a aumentar su existencia desarrollando operaciones de producción-venta de artículos, y aumentan la posibilidad de obtener tasas de rentabilidad más altas. 
No. 1

Enero - Junio 2015 ISSN 0122-820X

PP: 16-29
Las empresas han adoptado el modelo de Responsabilidad Social Corporativa (RSC) para cumplir con los requisitos de la sustentabilidad, a través del cual se fortalece su cadena de valor y su relación con los colaboradores y asociados, con el fin de asegurar a las futuras generaciones una sustentabilidad adecuada [3]. Según [1], señalan que para que la empresa sea viable necesita centrar su atención en dos conceptos: rentabilidad e imagen. De esta manera el concepto de sustentabilidad se valida en una visión interna que está dada por lo que los propietarios de la empresa esperan de ella, en este caso identificada por la rentabilidad y por una visión externa de lo que el conjunto de la comunidad espera de la empresa que se identifica como imagen. Según [4] la sustentabilidad debe considerarse como un instrumento para viabilizar el sistema económico y social en un horizonte temporal y espacial dado, de tal manera que permita la perdurabilidad de las empresas y la supervivencia del ser humano mediante una vida saludable, segura, productiva y en armonía con la naturaleza y los valores espirituales.

\subsection{La acreditación de la sustentabilidad}

Incluso las Bolsas de Valores han tomado cartas en el asunto para participar en el proceso de acreditar a las empresas que cumplen con los requisitos de sustentabilidad. "En la última década del siglo XX, Europa comenzó a buscar respuestas a partir de los gobiernos para promover la implicación moral y política del sector privado con la sustentabilidad a partir de un alto desempleo de larga duración y una crisis de exclusión social. En ese continente se establecieron regulaciones estatales muy fuertes en materia de RSE que, junto con las acciones de organizaciones como Global Reporting Initiative, impulsaron el desarrollo de reportes para la rendición de cuentas de las empresas en su totalidad de sus stakeholders (grupos de interés)" [1].
“También en Estados Unidos se vino trabajando de forma continuada durante las últimas décadas y se crearon organismos como el Business for Social Responsibility (BSR) y el World Business Council for Sustainable Development (WBCSD), que junto con los lineamientos de Global Reporting Initiative (GRI) (s.f.) impulsaron la publicación voluntaria de informes sociales y ambientales" [1]. "Como resultado, la responsabilidad social corporativa (RSC) ha emergido como una prioridad ineludible para los líderes de los negocios de todos los países" [5].

\subsection{El sector industrial en el índice sustentable}

En diciembre de 2011 la Bolsa Mexicana de Valores inició el funcionamiento del índice de Precios y Cotizaciones (IPC) Sustentable, que incorpora a las compañías cotizantes y que destacan en materia de sustentabilidad de acuerdo a los principios definidos en el Pacto Global de la ONU, donde se evalúan tres pilares: (i) manejo y uso de recursos naturales, (ii) responsabilidad social con sus principales grupos de interés (empleados, proveedores, clientes y comunidad en que se desempeñan y (iii) gobierno corporativo. Las empresas son evaluadas por calificadores independientes expertos en la materia, las empresas que desarrollan la calificación son, Ecovalores representante en México de EIRIS empresa inglesa que analiza empresas listadas en diferentes bolsas de valores alrededor del mundo y la Universidad Anáhuac del Sur.

El deterioro ambiental actual, resultado de la actividad industrial y de la explosión demográfica en los dos últimos siglos, pone a la sociedad en una situación en la que se deben replantear los procesos de producción bajo una óptica del máximo aprovechamiento de energía y recursos naturales [1], [6], en el IPC sustentable de la Bolsa Mexicana de Valores se encuentran siete empresas que 
pertenecen al sector industrial.

\subsection{Estudios empíricos}

Existen varios estudios empíricos sobre el efecto en la rentabilidad de las empresas que desarrollan actividades de gestión ambiental o son responsables socialmente [7], [8], [9], [10].

En[7]seencontrarondiferentes investigaciones que han buscado contrastar si la gestión ambiental corporativa tiene efectos sobre el desempeño financiero donde los resultados no son unánimes, pues mientras algunos autores consideran que la gestión ambiental beneficia a la empresa, otros concluyen que perjudica a su situación financiera, existiendo también estudios que consideran que tal relación es nula. Estos mismos autores [7], hicieron además un estudio empírico en la Bolsa de Valores de España, donde hallaron que el mercado accionario español reacciona de forma diferente ante noticias ambientales relacionadas con gastos e inversiones realizadas por las empresas cotizadas, dependiendo de si la situación económica del país se encuentra en fase de bonanza o bien de crisis, hay una relación positiva en época de bonanza y negativa cuando es una época de crisis, para esta investigación usaron las noticias de bases de datos de la firma Dow Jones y de los principales diarios españoles de acuerdo con una metodología de eventos.

Por otra parte en [8] se analizaron la relación entre el comportamiento social de la empresa y sus resultados financieros, explican que después de 30 años y 95 estudios empíricos, la relación entre el comportamiento social empresarial y los resultados financieros sigue siendo confusa La relación entre la empresa social y sus rendimientos financieros, fue estudiada por [9] a través de un proceso de meta-análisis de 52 estudios cuantitativos que muestran que a pesar de existir una correlación positiva de los resultados financieros de las empresas cuando asumen un comportamiento social la relación tiende a ser bidireccional y simultánea, por ello aún no se pueden lograr conclusiones generalizables.

En [10] se hizo un estudio con 19 compañías líderes del sector del índice Dow Jones Sustainability y evaluó el comportamiento de los ratios financieros. En cuanto al margen operativo y el margen neto las empresas sustentables muestran un comportamiento superior a sus respectivas industrias, en el caso del rendimiento sobre los activos (ROA) tienen un valor muy similar entre las empresas del índice sustentable y sus industrias, las compañías líderes en sostenibilidad muestran un ratio de $5.49 \%$ frente al $5.20 \%$ de sus respectivas industrias, en lo que se refiere al rendimiento del capital contable (ROE) muestran un ratio mayor que las respectivas industrias. En [11] se encontraron que las empresas del índice responsable español presentan un rendimiento sobre el capital contable de $16.27 \%$ superior al $16.25 \%$ de las empresas de otros índices, pero no existen diferencia estadísticamente significativa.

El objetivo de esta investigación es probar empíricamente la acreditación de sustentabilidad y su efecto en los ratios de rentabilidad en las empresas del sector industrial que cotizan en la BMV versus las que no se certificaron.

\section{Materiales y métodos}

Las empresas que ingresan al índice sustentable de la Bolsa Mexicana de Valores, obtienen la acreditación en tres áreas (manejo y uso de recursos naturales, responsabilidad social con sus principales grupos de interés y gobierno corporativo), que deben reflejarse en mayores márgenes de rentabilidad como resultado de los beneficios que debieran obtener por la acreditación, en la medida que se vuelven más eficientes por gastar menos agua y energía, generar menos basura, logrando ahorros importantes que se reflejan en la reducción de costos y evitando problemas por atender
Enero - Junio 2015 ISSN 0122-820X PP: 16-29

\author{
(1)
}


No. 1

Enero - Junio 2015

ISSN 0122-820X

PP: $16-29$ adecuadamente a los diferentes grupos de interés en las empresas.

Los resultados de los estudios empíricos sobre la relación que existe entre los márgenes de rentabilidad y la acreditación que obtienen estas empresas para ingresar a los índices sustentables de las bolsas de valores son contradictorios, dado que la acreditación como empresas sustentables no siempre aseguran mayores márgenes de rendimiento según los resultados de las investigaciones de en [8] y [11], y no existe un estudio empírico sobre la acreditación de las empresas para ingresar el IPC sustentable de la Bolsa Mexicana de Valores y su efecto en la rentabilidad, por ello se plantean las siguientes preguntas.

¿Cuál es el efecto en la rentabilidad por haberse acreditado en las áreas de manejo y uso de recursos naturales, responsabilidad social y gobierno corporativo para ingresar al índice sustentable de la Bolsa Mexicana de Valores, en las empresas del sector industrial versus las que no se certificaron?

Las empresas que obtienen la acreditación requerida por el índice bursátil de la Bolsa Mexicana de Valores debieran mostrar reducciones en los costos y con ello mayores beneficios en la rentabilidad, donde los ratios que miden los márgenes de rentabilidad tendrían que mostrar cambios significativamente estadísticos con valores de rentabilidad mayores comparados con las empresas que no obtuvieron la acreditación, para lo cual se plantean las hipótesis de trabajo, donde $\mathrm{H}_{0} \mathrm{y}_{1}$ es la hipótesis nula y la hipótesis alternativa respectivamente:

\section{Hipótesis principal}

$\mathbf{H}_{0}$. No existe diferencia estadística en los valores de los ratios que miden la rentabilidad de las empresas industriales que cotizan en el índice sustentable de la BMV versus las que no están en el índice sustentable.
$\mathbf{H}_{1}$ Existe diferencia estadística en los valores de los ratios que miden la rentabilidad de las empresas industriales que cotizan en el índice sustentable de la BMV versus las que no están en el índice sustentable.

Por otra parte debiera esperarse que las empresas que obtuvieron la acreditación exigida por los índices bursátiles denominados sustentables, tuvieran que mostrar mayores márgenes de rentabilidad después de ingresar al índice bursátil sustentable, por ello se plantea la siguiente hipótesis.

Hipótesis secundaria

$\mathbf{H}_{\mathbf{0}}$ No existe diferencia estadística en los valores de los ratios que miden la rentabilidad de las empresas industriales que cotizan en la BMV antes versus después de incorporarse al IPC sustentable.

$\mathbf{H}_{1}$ Existe diferencia estadística en los valores de los ratios que miden la rentabilidad de las empresas industriales que cotizan en la BMV antes versus después de incorporarse al IPC sustentable.

\subsection{Materiales}

Se estudiaron las 30 empresas del sector industrial que cotizan en la Bolsa Mexicana de Valores, las cuales se muestran en la Tabla I, donde 7 empresas ingresaron al índice sustentable de la Bolsa Mexicana de Valores en el año 2011. 
Tabla I. Empresas del IPC sustentable de la Bolsa Mexicana de Valores

\begin{tabular}{|c|c|c|}
\hline Núm. & Clave de cotización & Razón social \\
\hline 1 & ACCELSA & ACCEL, S.A.B. DE C.V. \\
\hline 2 & AEROMEX & GRUPO AEROMÉXICO S.A.B. DE C.V. \\
\hline 3 & $* * * A L F A$ & ALFA, S.A.B. DE C.V. \\
\hline 4 & ARA & CONSORCIO ARA, S.A.B. DE C.V. \\
\hline 5 & ARISTOS & CONSORCIO ARISTOS, S.A.B. DE C.V. \\
\hline 6 & $* * *$ ASUR & GRUPO AEROPORTUARIO DEL SURESTE, S.A.B. DE C.V. \\
\hline 7 & CERAMIC & INTERNACIONAL DE CERAMICA, S.A.B. DE C.V. \\
\hline 8 & DINE & DINE, S.A.B. DE C.V. \\
\hline 9 & GAP & GRUPO AEROPORTUARIO DEL PACIFICO, S.A.B. DE C.V. \\
\hline 10 & GCARSO & GRUPO CARSO, S.A.B. DE C.V. \\
\hline 11 & $* * * \mathrm{GEO}$ & CORPORACIÓN GEO, S.A.B. DE C.V. \\
\hline 12 & GISSA & GRUPO INDUSTRIAL SALTILLO, S.A.B. DE C.V. \\
\hline 13 & GMD & GRUPO MEXICANO DE DESARROLLO, S.A.B. DE C.V. \\
\hline 14 & GSANBOR & GRUPO SANBORNS, S.A.B. DE C.V. \\
\hline 15 & HOGAR & CONSORCIO HOGAR, S.A.B. DE C.V. \\
\hline 16 & ***HOMEX & DESARROLLADORA HOMEX, S.A.B. DE C.V. \\
\hline 17 & $* * * \mathrm{ICA}$ & EMPRESAS ICA, S.A.B. DE C.V. \\
\hline 18 & IDEAL & IMPULSORA DEL DESARROLLO Y EL EMPLEO EN AMÉRICA LATINA, S.A.B. DE C.V. \\
\hline 19 & INCARSO & INMUEBLES CARSO, S.A.B. DE C.V. \\
\hline 20 & KUO & GRUPO KUO, S.A.B. DE C.V. \\
\hline 21 & LAMOSA & GRUPO LAMOSA S.A.B. DE C.V. \\
\hline 22 & OHLMEX & OHL MÉXICO, S.A.B. DE C.V. \\
\hline 23 & $* * * O M A$ & GRUPO AEROPORTUARIO DEL CENTRO DEL NORTE S.A.B. DE C.V. \\
\hline 24 & PASA & PROMOTORA AMBIENTAL, S.A.B. DE C.V. \\
\hline 25 & PINFRA & PROMOTORA Y OPERADORA DE INFRAESTRUCTURA S.A.B. DE C.V. \\
\hline 26 & SARE & SARE HOLDING S.A.B. DE C.V. \\
\hline 27 & TMM & GRUPO TMM, S.A. \\
\hline 28 & $* * * U R B I$ & URBI DESARROLLOS URBANOS, S.A.B. DE C.V. \\
\hline 29 & VESTA & CORPORACIÓN INMOBILIARIA S.A.B. DE C.V. \\
\hline 30 & VOLAR & CONTROLADORA VUELA COMPAÑÍA DE AVIACIÓN, S.A.B DE C.V. \\
\hline
\end{tabular}

Un ratio es el coeficiente de la relación que existe entre dos rubros de los estados financieros y se usan para medir el desempeño empresarial en las áreas de la empresa. Para el cálculo de los ratios que miden los márgenes de rentabilidad se utilizaron los estados financieros de las 30 empresas que integran el IPC, la información se obtuvo de la base de datos de Economatica y de la Bolsa Mexicana de Valores, con los cuales se determinaron 6 ratios financieros, que se presentan en la
Tabla II, por el periodo que comprende del cuarto trimestre del 2009 al primer trimestre del 2014 para cada una de las empresas, en total se analizaron 540 trimestres de información financiera, que equivale a 3240 ratios financieros.

La información se separó en dos grupos: (i) las empresas que se acreditaron para ingresar al índice sustentable de la BMV, y (ii) las empresas del sector industrial que no han 
No. 1

obtenido la acreditación de sustentable, con la finalidad de tener dos grupos de empresas y comparar los valores de los ratios de rentabilidad en las empresas certificadas como sustentables con aquellas que no obtuvieron la acreditación se sustentabilidad, que en este caso se considera el grupo control.

Posteriormente el grupo de empresas que obtuvo su acreditación para ingresar al índice sustentable se separó en dos periodos de tiempo, el cuarto trimestre de 2009 hasta el cuarto trimestre de 2011 y el segundo de primer trimestre de 2012 hasta el primer trimestre de 2014. El motivo de lo anterior fue para tener dos periodos de 9 trimestres cada uno y comparar cual era el comportamiento de los ratios de rentabilidad antes y después de diciembre de 2011, fecha en que las empresas obtuvieron su acreditación para incorporarse al índice sustentable del IPC de la Bolsa Mexicana de Valores.

Tabla II. Ratios financieros

\begin{tabular}{|c|c|l|}
\hline $\begin{array}{l}\text { Ratio } \\
\text { financiero }\end{array}$ & Fórmula & \multicolumn{1}{c|}{ Interpretación } \\
\hline 1 & $\begin{array}{c}\text { Resultado neto } \\
\text { Ventas netas }\end{array}$ & $\begin{array}{l}\text { Margen de utilidad neta sobre las ventas. Indica el porcentaje de utilidad neta respecto a las ventas netas. Entre } \\
\text { más alto es el valor del ratio, hay mayor utilidad por cada peso de ventas. }\end{array}$ \\
\hline 2 & $\begin{array}{c}\text { Resultado neto } \\
\text { Activo total }\end{array}$ & $\begin{array}{l}\text { Cuantifica la rentabilidad del activo o inversión total, también se le conoce como ROA (por sus siglas en inglés } \\
\text { Return on Assets). }\end{array}$ \\
\hline 3 & $\begin{array}{c}\text { Resultado neto } \\
\text { Capital contable }\end{array}$ & $\begin{array}{l}\text { Evalúa la rentabilidad de los accionistas. También se le conoce como ROE (por sus siglas en ingles Return } \\
\text { on equity). }\end{array}$ \\
\hline 4 & $\begin{array}{l}\text { Resultado bruto } \\
\text { Ventas netas }\end{array}$ & $\begin{array}{l}\text { Mide el margen de utilidad bruta, con relación a las ventas, entre más alto es el coeficiente, menores son los } \\
\text { costos de venta. }\end{array}$ \\
\hline 5 & Resultado de operación & $\begin{array}{l}\text { Cuantifica el margen de utilidad de operación sobre ventas, entre más alto es el valor del ratio, significa me- } \\
\text { nores costos de venta y gastos de operación. }\end{array}$ \\
\hline 6 & Rentas netas & $\begin{array}{l}\text { Mide el margen de utilidad antes de impuestos sobre las ventas. Entre más alto es el coeficiente significa que } \\
\text { los costos de venta, gastos de operación y financieros se han reducido. }\end{array}$ \\
\hline
\end{tabular}

Fuente: Autor con base en [12], [13], [14], [15].

\subsection{Métodos}

Para evaluar el efecto en la rentabilidad de las empresas que se acreditaron como sustentables, primero se calculó la diferencia de entre las medias de los valores de los ratios de rentabilidad entre las empresas que se acreditaron como sustentables versus las que no se certificaron, y después se calculó la diferencia entre las medias de los valores de los ratios de rentabilidad del periodo 2009-2011 versus el periodo de 2012-2014, para evaluar el efecto en la rentabilidad de las empresas que se acreditaron como sustentables y comparar sus márgenes de rentabilidad antes y después de certificarse.

Para conocer la diferencia entre los valores promedios, primero se calcula la diferencia entrecadaparde valores yse determinalamedia y la desviación estándar $S_{D}$ de las diferencias de medias muestrales, posteriormente se calcula el intervalo de confianza del $95 \%$ de dos colas para la diferencia del valor medio, con las formulas:

$\bar{D}=\frac{\sum D_{i}}{n} \quad S_{D}=\sqrt{\frac{\sum D_{i}^{2} n \bar{D}^{2}}{n-1}} \quad \bar{D} \pm t \propto / 2 \frac{S_{D}}{\sqrt{N}}$

Dónde:

$S_{D}=$ Desviación estándar de la diferencia de medias de dos grupos

$D^{2}=$ Cuadrado de la diferencia apareada

$n=$ Número de pares muestrales apareados 
$\bar{D}=$ Media de las diferencias apareadas

$$
\mu=\text { Media del grupo " } i \text { " }
$$

Debido a que el error estándar de la diferencia media se calcula con base en la desviación estándar de la muestra de diferencias, y ya que en general se puede suponer que los valores $\mathrm{D}$ tienen una distribución normal, la distribución apropiada para probar la hipótesis nula es la distribución $t$.

\section{Resultados y análisis}

Los resultados de la investigación se presentan en las Tablas III, IV, V, VI, VII, VIII Y IX, de los cuales se hace la interpretación estadística financiera para así contestar las preguntas de investigación y validar las hipótesis, correlacionando los resultados con otros similares. En la Tabla III se observa que los valores en los seis ratios de márgenes de rentabilidad en sus distintas modalidades en el caso de las empresas acreditadas como sustentables tienen valores más altos que las empresas no acreditadas, aunque solo en cuatro existen diferencia estadística significativa, los cuales son: margen de utilidad neta sobre ventas, rentabilidad del capital contable, margen de utilidad de operación y margen de utilidad antes de impuestos sobre las ventas. Estos resultados demuestran que las empresas del sector industrial acreditadas como sustentables si tienen diferentes valores en su rentabilidad frente a aquellas empresas que no se han acreditado.

Cuando se analizan los promedios de los ratios de rentabilidad de las 7 empresas que se acreditaron para ingresar al índice sustentable de la BMV los resultados fueron contradictorios, como se muestra en la Tabla IV, en los valores promedio de los ratios de margen de utilidad sobre ventas, rendimiento del activo y rendimiento del capital contable sus valores disminuyeron después de acreditarse; en cambio en los valores de los ratios de margen de utilidad bruta, margen de utilidad de operación, y margen de utilidad antes de impuestos los valores promedio si aumentaron después de que las empresas se acreditaron, de estos ratios que aumentaron solo dos de ellos tienen diferencia estadística significativa, el ratio de margen de utilidad bruta y el de margen de utilidad de operación.

En el caso de las empresas que no se acreditaron como sustentables, al analizar la información financiera se encontró que TMM y SARE desde el tercer trimestre del 2009 presentan perdidas, reflejándose en la rentabilidad, su giro es la construcción, donde se presume que la tendencia del sector ha afectado sus resultados negativamente.

Tabla III. Valor de los ratios de rentabilidad de las empresas certificadas como sustentables versus las no certificadas

\begin{tabular}{|c|c|c|c|c|c|c|c|c|c|c|}
\hline \multirow[b]{2}{*}{ No. } & \multirow[b]{2}{*}{ Ratio } & \multicolumn{2}{|c|}{ Promedio ratios rentabilidad } & \multirow{2}{*}{$\begin{array}{c}\text { Media de la } \\
\text { diferencia } \\
\text { apareada }\end{array}$} & \multirow{2}{*}{\multicolumn{2}{|c|}{ Intervalo de confianza }} & \multirow[b]{2}{*}{$\begin{array}{l}\text { Limite } \\
\text { inferior }\end{array}$} & \multirow[b]{2}{*}{$\begin{array}{l}\text { Limite } \\
\text { superior }\end{array}$} & \multirow{2}{*}{$\begin{array}{c}\text { Valor } \\
\text { calculado de } \\
\text { la estadistica } \\
\text { "t" de prueba }\end{array}$} & \multirow[b]{2}{*}{$\begin{array}{l}\text { Validación de } \\
\text { la hipótesis }\end{array}$} \\
\hline & & $\begin{array}{c}\text { Certificadas } \\
\text { como } \\
\text { sustentables } \\
2012-2014\end{array}$ & $\begin{array}{c}\text { No Sustentables } \\
\text { 2012-2014 }\end{array}$ & & & & & & & \\
\hline 1 & Resultado Neto $\div$ Ventas Netas & 0.1117 & 0.0156 & 0.0961 & \pm & 0.0834 & 0.0127 & 0.1795 & 2.2584 & Rechaza \\
\hline 2 & Resultado Neto $\div$ Activo Total & 0.0358 & 0.0245 & 0.0113 & \pm & 0.0168 & -0.0056 & 0.0281 & 1.3115 & Acepta \\
\hline 3 & Resultado Neto $\div$ Capital Contable & 0.1078 & -0.2949 & 0.4027 & \pm & 0.2690 & 0.1337 & 0.6717 & 2.9340 & Rechaza \\
\hline 4 & Resultado Bruto $\div$ Ventas Netas & 0.4171 & 0.3892 & 0.0279 & \pm & 0.0354 & -0.0075 & 0.0633 & 1.5449 & Acepta \\
\hline 5 & Resultado Operación $\div$ Ventas Netas & 0.2245 & 0.0779 & 0.1466 & \pm & 0.0871 & 0.0595 & 0.2337 & 3.2997 & Rechaza \\
\hline 6 & Resultado antes de impuestos $\div$ Ventas Netas & 0.1905 & 0.0276 & 0.1630 & \pm & 0.0827 & 0.0802 & 0.2457 & 3.8605 & Rechaza \\
\hline
\end{tabular}


Respuestas

Cúcuta-Colombia

Vol. 20

No. 1

Enero - Junio 2015

ISSN 0122-820X

PP: $16-29$
La acreditación de sustentabilidad y su impacto en los márgenes de rentabilidad del sector industrial de la Bolsa Mexicana de Valores

Tabla IV. Valor de los ratios de rentabilidad de las empresas del sector industrial antes versus después de ingresar al IPC sustentable.

\begin{tabular}{|c|c|c|c|c|c|c|c|c|c|c|}
\hline \multirow{2}{*}{ No } & \multirow{2}{*}{ Ratio } & \multicolumn{2}{|c|}{$\begin{array}{c}\text { Promedio ratios } \\
\text { rentabilidad }\end{array}$} & \multirow{2}{*}{$\begin{array}{c}\text { Media de la } \\
\text { diferencia } \\
\text { apareada }\end{array}$} & \multirow{2}{*}{\multicolumn{2}{|c|}{$\begin{array}{l}\text { Intervalo de } \\
\text { confianza }\end{array}$}} & \multirow{2}{*}{$\begin{array}{l}\text { Limite } \\
\text { inferior }\end{array}$} & \multirow{2}{*}{$\begin{array}{l}\text { Limite } \\
\text { superior }\end{array}$} & \multirow{2}{*}{$\begin{array}{c}\text { Valor } \\
\text { calculado } \\
\text { de la } \\
\text { estadistica } \\
\text { "t" de } \\
\text { prueba } \\
\end{array}$} & \multirow{2}{*}{$\begin{array}{c}\text { Validación } \\
\text { de la } \\
\text { hipótesis }\end{array}$} \\
\hline & & 2009-2011 & 2012-2014 & & & & & & & \\
\hline & & & & & & & & & & \\
\hline 1 & Resultado Neto $\div$ Ventas Netas & 0.1206 & 0.1117 & 0.0089 & \pm & 0.0570 & -0.0481 & 0.0659 & 0.3064 & Acepta \\
\hline 2 & Resultado Neto $\div$ Activo Total & 0.0450 & 0.0358 & 0.0092 & \pm & 0.0224 & -0.0131 & 0.0316 & 0.8091 & Acepta \\
\hline 3 & Resultado Neto $\div$ Capital Contable & 0.1172 & 0.1078 & 0.0094 & \pm & 0.0149 & -0.0056 & 0.0243 & 1.2293 & Acepta \\
\hline 4 & Resultado Bruto $\div$ Ventas Netas & 0.3702 & 0.4171 & -0.0469 & \pm & 0.0275 & -0.0744 & -0.0194 & -3.3404 & Rechaza \\
\hline 5 & Resultado Operación $\div$ Ventas Netas & 0.1896 & 0.2245 & -0.0349 & \pm & 0.0250 & -0.0599 & -0.0099 & -2.7405 & Rechaza \\
\hline 6 & Resultado antes de impuestos $\div$ Ventas Netas & 0.1699 & 0.1905 & -0.0207 & \pm & 0.0214 & -0.0420 & 0.0007 & -1.8942 & Acepta \\
\hline
\end{tabular}

Fuente: Autor

En la Tabla V se presentan los valores de los ratios del margen de utilidad bruta, y se observa que ICA y OMA aumentan en su valor después de certificarse para ingresar al IPC sustentable de la BMV, las otras empresas disminuyen en sus márgenes de utilidad bruta, de las empresas que mostraron aumentos solo OMA tuvo cambio estadísticamente significativo.

Tabla V. Margen de utilidad bruta en las empresas del sector industrial antes y después de ingresar al índice sustentable.

\begin{tabular}{|c|c|c|c|c|c|c|c|c|c|c|}
\hline \multirow[t]{2}{*}{ No. } & \multirow[t]{2}{*}{ Empresa } & \multicolumn{2}{|c|}{$\begin{array}{l}\text { Promedio ratio } \\
\text { Resultado Bruto } \div \\
\text { Ventas Netas }\end{array}$} & \multirow[t]{2}{*}{$\begin{array}{c}\text { Media de la } \\
\text { diferencia } \\
\text { apareada }\end{array}$} & \multicolumn{2}{|c|}{$\begin{array}{c}\text { Intervalo de } \\
\text { confianza }\end{array}$} & \multirow[t]{2}{*}{$\begin{array}{l}\text { Limite } \\
\text { inferior }\end{array}$} & \multirow[t]{2}{*}{$\begin{array}{l}\text { Limite } \\
\text { superior }\end{array}$} & \multirow{2}{*}{$\begin{array}{c}\text { Valor } \\
\text { calculado } \\
\text { de la } \\
\text { estadistica } \\
\text { "t" de } \\
\text { prueba }\end{array}$} & \multirow[t]{2}{*}{$\begin{array}{l}\text { Validación } \\
\text { de la } \\
\text { hipótesis }\end{array}$} \\
\hline & & 2009-2011 & 2012-2014 & & & & & & & \\
\hline 1 & ALFA & 0.1914 & 0.1783 & 0.0131 & \pm & 0.0073 & 0.0058 & 0.0204 & 3.5202 & Rechaza \\
\hline 2 & ASUR & 0.9849 & 0.9819 & 0.0029 & \pm & 0.0009 & 0.0021 & 0.0038 & 6.7152 & Rechaza \\
\hline 3 & GEO & 0.2992 & 0.1736 & 0.1256 & \pm & 0.1188 & 0.0068 & 0.2443 & 2.0721 & Rechaza \\
\hline 4 & HOMEX & 0.2946 & -0.0023 & 0.2969 & \pm & 0.2727 & 0.0242 & 0.5696 & 2.1336 & Rechaza \\
\hline 5 & ICA & 0.1479 & 0.1668 & -0.0189 & \pm & 0.0199 & -0.0388 & 0.0010 & -1.8644 & Acepta \\
\hline 6 & OMA & 0.3855 & 0.5071 & -0.1216 & \pm & 0.0376 & -0.1592 & -0.0840 & -6.3425 & Rechaza \\
\hline 7 & URBI & 0.2882 & 0.1557 & 0.1325 & \pm & 0.0921 & 0.0404 & 0.2246 & 2.8189 & Rechaza \\
\hline
\end{tabular}

Fuente: Autor

En el análisis de los márgenes de utilidad de operación como se muestra en la Tabla VI, solo ALFA, ASUR, ICA y OMA tienen aumentos después de ingresar al índice sustentable de la BMV y las otras empresas muestran disminuciones, es decir, el 50\% de las empresas acreditadas tuvieron aumentos en el margen de utilidad de operación y de estas empresas solo ALFA e ICA tuvieron cambios estadísticamente significativos.

Tabla VI. Margen de utilidad de operación en las empresas del sector industrial antes y después de ingresar al índice sustentable.

\begin{tabular}{|c|c|c|c|c|c|c|c|c|c|c|}
\hline \multirow[t]{2}{*}{ No. } & \multirow[t]{2}{*}{ Empresa } & \multicolumn{2}{|c|}{$\begin{array}{c}\text { Promedio ratio } \\
\text { Resultado Operación } \div \\
\text { Ventas Netas }\end{array}$} & \multirow{2}{*}{$\begin{array}{c}\text { Media de la } \\
\text { diferencia } \\
\text { apareada }\end{array}$} & \multirow{2}{*}{\multicolumn{2}{|c|}{$\begin{array}{c}\text { Intervalo de } \\
\text { confianza }\end{array}$}} & \multirow[t]{2}{*}{$\begin{array}{l}\text { Limite } \\
\text { inferior }\end{array}$} & \multirow[t]{2}{*}{$\begin{array}{l}\text { Limite } \\
\text { superior }\end{array}$} & \multirow{2}{*}{\begin{tabular}{|c|} 
Valor \\
calculado \\
de la \\
estadistica \\
"t" de \\
prueba
\end{tabular}} & \multirow{2}{*}{$\begin{array}{l}\text { Validación } \\
\text { de la } \\
\text { hipótesis }\end{array}$} \\
\hline & & 2009-2011 & 2012-2014 & & & & & & & \\
\hline 1 & ALFA & 0.0740 & 0.0744 & -0.0003 & \pm & 0.0058 & -0.0061 & 0.0055 & -0.1326 & Acepta \\
\hline 2 & ASUR & 0.4314 & 0.5010 & -0.0696 & \pm & 0.0021 & -0.0717 & -0.0675 & -6.7213 & Rechaza \\
\hline 3 & GEO & 0.1591 & 0.0850 & 0.0741 & \pm & 0.0068 & 0.0673 & 0.0809 & 2.7120 & Rechaza \\
\hline 4 & HOMEX & 0.1457 & -0.1555 & 0.3011 & \pm & 0.0242 & 0.2770 & 0.3253 & 1.5860 & Acepta \\
\hline 5 & ICA & 0.0851 & 0.0862 & -0.0011 & \pm & -0.0388 & 0.0377 & -0.0399 & -0.0913 & Acepta \\
\hline 6 & OMA & 0.2379 & 0.3634 & -0.1255 & \pm & -0.1592 & 0.0337 & -0.2847 & -6.7649 & Rechaza \\
\hline 7 & URBI & 0.2044 & 0.0729 & 0.1315 & \pm & 0.0404 & 0.0912 & 0.1719 & 4.3585 & Rechaza \\
\hline
\end{tabular}


En la Tabla VII se presentan los valores promedio de los ratios de margen de utilidad antes de impuestos sobre las ventas, donde ALFA, ASUR y OMA muestran incrementos después de ingresar al índice sustentable de la BMV, es decir, tan solo $42.85 \%$ de las empresas que se acreditaron como sustentables, en las demás empresas disminuyen los valores de estos ratios, en las compañías que aumentaron en este ratio solo ASUR y OMA tienen cambios estadísticamente significativos.

Tabla VII. Margen de utilidad antes de impuestos en las empresas del sector industrial antes y después de ingresar al índice sustentable.

\begin{tabular}{|c|c|c|c|c|c|c|c|c|c|c|}
\hline \multirow[t]{2}{*}{ No. } & \multirow[t]{2}{*}{ Empresa } & \multicolumn{2}{|c|}{$\begin{array}{c}\text { Promedio ratio } \\
\text { Resultado antes de } \\
\text { impuestos } \div \text { Ventas } \\
\text { Netas }\end{array}$} & \multirow[t]{2}{*}{$\begin{array}{c}\text { Media de la } \\
\text { diferencia } \\
\text { apareada }\end{array}$} & \multirow{2}{*}{\multicolumn{2}{|c|}{$\begin{array}{c}\text { Intervalo de } \\
\text { confianza }\end{array}$}} & \multirow[t]{2}{*}{$\begin{array}{l}\text { Limite } \\
\text { inferior }\end{array}$} & \multirow[t]{2}{*}{$\begin{array}{l}\text { Limite } \\
\text { superior }\end{array}$} & \multirow{2}{*}{\begin{tabular}{|c|} 
Valor \\
calculado \\
de la \\
estadistica \\
"t" de \\
prueba \\
\end{tabular}} & \multirow[t]{2}{*}{$\begin{array}{c}\text { Validación } \\
\text { de la } \\
\text { hipótesis }\end{array}$} \\
\hline & & 2009-2001 & 2012-2014 & & & & & & & \\
\hline 1 & ALFA & 0.0535 & 0.0547 & -0.0012 & \pm & 0.0204 & -0.0216 & 0.0191 & -0.2420 & Acepta \\
\hline 2 & ASUR & 0.4360 & 0.4986 & -0.0626 & \pm & 0.0038 & -0.0664 & -0.0588 & -8.2605 & Rechaza \\
\hline 3 & GEO & 0.1212 & 0.0533 & 0.0680 & \pm & 0.2443 & -0.1764 & 0.3123 & 4.1293 & Rechaza \\
\hline 4 & HOMEX & 0.1314 & -0.1804 & 0.3118 & \pm & 0.5696 & -0.2578 & 0.8814 & 1.6048 & Acepta \\
\hline 5 & ICA & 0.0477 & 0.0127 & 0.0350 & \pm & 0.0010 & 0.0341 & 0.0360 & 3.6957 & Rechaza \\
\hline 6 & OMA & 0.2123 & 0.3338 & -0.1215 & \pm & -0.0840 & -0.0375 & -0.2056 & -6.2884 & Rechaza \\
\hline 7 & URBI & 0.1941 & 0.0483 & 0.1457 & \pm & 0.2246 & -0.0789 & 0.3703 & 3.3965 & Rechaza \\
\hline
\end{tabular}

Fuente: Autor

La Tabla VIII muestra que solo ASUR y OMA aumentaron el valor promedio de sus ratios de utilidad neta sobre ventas después de haberse acreditado como sustentable, las otras empresas disminuyeron en sus valores, es decir, solo el $28 \%$ de empresas incrementó este margen de utilidad. De las empresas que tuvieron aumentos solo ASUR y OMA tienen cambios estadísticamente significativos.

Tabla VIII. Margen de utilidad neta en las empresas del sector industrial antes y después de ingresar al índice sustentable.

\begin{tabular}{|c|c|c|c|c|c|c|c|c|c|c|}
\hline \multirow[t]{2}{*}{ No. } & \multirow[t]{2}{*}{ Empresa } & \multicolumn{2}{|c|}{$\begin{array}{l}\text { Promedio ratio } \\
\text { Resultado Neto } \div \\
\text { Ventas Netas }\end{array}$} & \multirow[t]{2}{*}{$\mid \begin{array}{c}\text { Media de la } \\
\text { diferencia } \\
\text { apareada }\end{array}$} & \multicolumn{2}{|c|}{$\begin{array}{l}\text { Intervalo de } \\
\text { confianza }\end{array}$} & \multirow[t]{2}{*}{$\begin{array}{l}\text { Limite } \\
\text { inferior }\end{array}$} & \multirow[t]{2}{*}{$\begin{array}{l}\text { Limite } \\
\text { superior }\end{array}$} & \multirow{2}{*}{$\begin{array}{c}\text { Valor } \\
\text { calculado } \\
\text { de la } \\
\text { estadistica } \\
\text { "t" de } \\
\text { prueba }\end{array}$} & \multirow[t]{2}{*}{$\begin{array}{c}\text { Validación } \\
\text { de la } \\
\text { hipótesis }\end{array}$} \\
\hline & & \begin{tabular}{|l|}
$2009-2011$ \\
\end{tabular} & 2012-2014 & & & & & & & \\
\hline & & & & & & & & & & \\
\hline 1 & ALFA & 0.0352 & 0.0343 & 0.0009 & \pm & 0.0071 & -0.0062 & 0.0081 & 0.2616 & Acepta \\
\hline 2 & ASUR & 0.3036 & 0.3996 & -0.0961 & \pm & 0.0167 & -0.1127 & -0.0794 & -11.3100 & Rechaza \\
\hline 3 & GEO & 0.0740 & 0.0302 & 0.0438 & \pm & 0.0182 & 0.0256 & 0.0620 & 4.7181 & Rechaza \\
\hline 4 & HOMEX & 0.0791 & -0.1429 & 0.2219 & \pm & 0.2703 & -0.0484 & 0.4923 & 1.6093 & Acepta \\
\hline 5 & ICA & 0.0262 & 0.0174 & 0.0088 & \pm & 0.0175 & -0.0087 & 0.0264 & 0.9863 & Acepta \\
\hline 6 & OMA & 0.2098 & 0.2811 & -0.0713 & \pm & 0.0203 & -0.0916 & -0.0510 & -6.8700 & Rechaza \\
\hline 7 & URBI & 0.1166 & 0.0329 & 0.0836 & \pm & 0.0616 & 0.0220 & 0.1453 & 2.6598 & Rechaza \\
\hline
\end{tabular}

En lo que se refiere al ratio de rentabilidad de la inversión, se observa en la Tabla IX, que solo ASUR y OMA mostraron incrementos después de haber ingresado al índice sustentable de la $\mathrm{BMV}$, es decir que solo $28 \%$ de las empresas y sus cambios fueron estadísticamente significativos. 
No. 1

Tabla IX. Rentabilidad del activo en las empresas del sector industrial antes y después de ingresar al índice sustentable.

\begin{tabular}{|c|c|c|c|c|c|c|c|c|c|c|}
\hline \multirow[t]{2}{*}{ No. } & \multirow[t]{2}{*}{ Empresa } & \multicolumn{2}{|c|}{$\begin{array}{c}\text { Promedio ratio de } \\
\text { Resultado Neto } \div \text { Activo } \\
\text { Total }\end{array}$} & \multirow{2}{*}{$\begin{array}{c}\text { Media de la } \\
\text { diferencia } \\
\text { apareada }\end{array}$} & \multirow{2}{*}{\multicolumn{2}{|c|}{$\begin{array}{c}\text { Intervalo de } \\
\text { confianza }\end{array}$}} & \multirow[t]{2}{*}{$\begin{array}{l}\text { Limite } \\
\text { inferior }\end{array}$} & \multirow[t]{2}{*}{$\begin{array}{l}\text { Limite } \\
\text { superior }\end{array}$} & \multirow{2}{*}{$\begin{array}{c}\text { Valor } \\
\text { calculado } \\
\text { de la } \\
\text { estadistica } \\
\text { "t" de } \\
\text { prueba }\end{array}$} & \multirow{2}{*}{$\begin{array}{l}\text { Validación } \\
\text { de la } \\
\text { hipótesis }\end{array}$} \\
\hline & & 2009-2001 & 2012-2014 & & & & & & & \\
\hline 1 & ALFA & 0.0426 & 0.0436 & -0.0010 & \pm & 0.0103 & -0.0113 & 0.0093 & -0.1897 & Acepta \\
\hline 2 & ASUR & 0.0662 & 0.1004 & -0.0342 & \pm & 0.0054 & -0.0396 & -0.0288 & -12.3833 & Rechaza \\
\hline 3 & GEO & 0.0468 & 0.0149 & 0.0319 & \pm & 0.0081 & 0.0238 & 0.0400 & 7.7463 & Rechaza \\
\hline 4 & HOMEX & 0.0436 & -0.0488 & 0.0924 & \pm & 0.1002 & -0.0079 & 0.1926 & 1.8065 & Acepta \\
\hline 5 & ICA & 0.0119 & 0.0067 & 0.0052 & \pm & 0.0069 & -0.0017 & 0.0121 & 1.4868 & Acepta \\
\hline 6 & OMA & 0.0562 & 0.0857 & -0.0294 & \pm & 0.0080 & -0.0374 & -0.0215 & -7.2303 & Rechaza \\
\hline 7 & URBI & 0.0476 & 0.0131 & 0.0346 & \pm & 0.0183 & 0.0163 & 0.0529 & 3.7139 & Rechaza \\
\hline
\end{tabular}

Fuente: Autor

En esta investigación se encontró que el valor promedio del ratio de rendimiento sobre los activos después de acreditarse como sustentables es de $3.58 \%$ frente a las empresas que no están certificadas que fue de $2.45 \%$ y en el rendimiento sobre el capital contable para las sustentables es de $10.78 \%$ frente a las no sustentables que es $-29.49 \%$ (véase Tabla III), en [10] encontró en las empresas sustentables del Dow Jones que los márgenes de rentabilidad del activo son de 5.49\% frente a las no sustentables de $5.20 \%$, y en el caso de la rentabilidad del capital contable fue de $16.27 \%$ en las sustentables frente al $16.25 \%$ en las no sustentables. Una de las aportaciones de esta investigación fue conocer los márgenes de rentabilidad de las empresas tanto sustentables como no sustentables de la $\mathrm{BMV}$, los cuales son menores a las empresas del índice sustentable Dow Jones estudiados en [10].

Cuando se compararon los valores de los ratios de rentabilidad de las empresas del sector industrial entre los periodos previos y posterior a la acreditación para ingresar al índice sustentable, los resultados entre los ratios son contradictorios (véase Tabla IV), tres ratios mostraron disminuciones, mientras los otros tres si tuvieron incrementos, estos resultados coinciden con [8], [9] en donde encontraron que a pesar de existir una correlación positiva de los resultados financieros de las empresas cuando asumen un comportamiento social la relación tiende a ser bidireccional y simultánea, por ello aún no se pueden lograr conclusiones generalizables.

Con los resultados empíricos de este trabajo se responde la pregunta principal de investigación, donde se encontró que los seis ratios de rentabilidad tienen valores más altos en las empresas que se acreditaron como sustentables frente a aquellas que no se acreditaron, las cuales tuvieron mayores márgenes de rentabilidad, pero no en todos ellos existe diferencia estadística significativa (véase Tabla III).

La hipótesis nula principal se rechaza parcialmente y se acepta en parte la hipótesis alternativa, porque se encontró que existe diferencia estadística en cuatro ratios, al contrastar los valores promedios de los ratios de rentabilidad de las empresas que se acreditaron para ingresar el IPC sustentable frente a las que no se acreditaron. En lo que se refiere a la hipótesis secundaria se encontró que al comparar los valores de los ratios financieros en los periodos previo y posterior a su ingreso al IPC sustentable de las empresas del sector industrial, se halló que cuatro de 
ellos disminuyeron (véase Tabla IV), por lo cual se rechaza la hipótesis nula secundaria parcialmente y se acepta en parte la hipótesis alternativa secundaria, dado que de los tres ratios que sí tuvieron incremento solo dos mostraron diferencia estadística significativa.

Los beneficios de acreditarse como sustentables a las industrias de la BMV se reflejan en mayores márgenes de utilidad bruta y de operación, pero se reducen en los márgenes de rentabilidad neta, (véase Tabla IV), lo cual sugiere que los intereses pagados por la financiación y la tasa fiscal deterioran las ventajas en los beneficios obtenidos. Sin embargo ocurre lo contrario en la investigación de [10], en donde se halló que en las empresas del índice sustentable del Dow Jones, tienen mayores márgenes de rentabilidad neta, estas compañías se caracterizan por mayor apetito para usar deuda, reflejándose en mayores tasas de rentabilidad neta sobre las ventas, es decir, que la financiación por deuda incrementa la rentabilidad cuando se certifican como sustentables. Estos resultados contradictorios entre las empresas del índice sustentable de la BMV y del Dow Jones, establecen la necesidad de investigar los factores que influyen en los bajos márgenes de rentabilidad neta en las empresas del sector industrial de la BMV que se certifican como sustentables.

Cuando se evalúa el comportamiento por cada uno de los ratios de rentabilidad y se contrastan los periodos antes y después de ingresar al IPC sustentable para cada una de las siete empresas del sector industrial certificadas como sustentables en la BMV (véase Tablas V, VI, VII, VIII y IX), se encontró que en el ratio de margen de utilidad bruta solo ICA, OMA y URBI mostraron aumentos en sus valores, y sólo en OMA y URBI sus diferencias fueron estadísticamente significativas; en el margen de utilidad de operación ALFA, ASUR, ICA y OMA mostraron aumentos en sus valores, y sólo en OMA y URBI fueron estadísticamente significativos; en lo que se refiere a los márgenes de utilidad antes de impuestos, las empresas que tuvieron incrementos fueron ALFA, ASUR y OMA y sólo en ASUR y OMA fueron estadísticamente significativos; en el margen de utilidad neta ALFA, ASUR y OMA aumentaron sus valores, y sólo en ASUR y OMA fueron estadísticamente significativos; en el ratio de rendimiento del activo ALFA, ASUR y OMA se incrementó, y sólo en ASUR y OMA fueron estadísticamente significativos. Las empresas ASUR y OMA tienen como giro fundamental la operación de la concesión de aeropuertos en México, de alguna manera tienen un mercado cautivo, lo cual asegura sus ingresos sean altos.

\section{Conclusiones}

Se contrastaron los valores de los ratios de rentabilidad de las empresas que se acreditaron como sustentables con las que no se certificaron, se encontró que los valores promedios de los ratios de las empresas sustentables son más altos, aunque solo existe diferencia estadística significativa en los ratios de margen de utilidad neta, rentabilidad del capital contable, margen de utilidad de operación y margen de utilidad antes de impuestos, con estos datos se contestó la pregunta principal de investigación $\mathrm{y}$ se probó la hipótesis principal, donde se aceptó la hipótesis alternativa parcialmente, porque si existe diferencia en cuatro ratios de rentabilidad de empresas que se acreditaron frente a las que no. Se rechaza parcialmente la hipótesis secundaria al comparar los valores de los ratios financieros de las empresas en los periodos previo y posterior a su ingreso al IPC sustentable, porque tres aumentaron y cuatro disminuyeron según Tabla IV.

Del análisis que se hizo por cada uno de los ratios de rentabilidad para todas las empresas certificadas como sustentables por los periodos previo y posterior a la acreditación de sustentabilidad, se encontró que OMA tiene incrementos en todos los ratios, ASUR en tres
Enero - Junio 2015

ISSN 0122-820X

PP: 16-29 
No. 1

Enero - Junio 2015 ISSN 0122-820X

PP: $16-29$ y URBI en dos, donde esos incrementos en los valores de los ratios fueron estadísticamente significativos.

Se encontró que los costos de ventas y los gastos de operación se reducen reflejándose en un incremento en los márgenes de rentabilidad, sin embargo en los valores de los ratios de margen de utilidad neta no se obtuvieron incrementos. Por lo cual hace necesario investigar los factores que afectan negativamente el margen de utilidad neta, entre ellos es necesario estudiar los gastos financieros que se pagan por los créditos que emplean las industrias, el cual podría ser un elemento de la gestión financiera que merme los márgenes de utilidad, otro de ellos podría ser la tendencia del sector en determinando lapso de tiempo.

Certificarse en las áreas de manejo y uso de recursos naturales, responsabilidad social y gobierno corporativo para ingresar al índice sustentable de las bolsas de valores si se refleja en aumento de márgenes de rentabilidad bruta y de operación en las empresas, pero hay que cuidar que la gestión financiera de las empresas contribuya a incrementar los márgenes de utilidad neta.

\section{Agradecimientos}

A la Escuela Superior de Comercio y Administración unidad Tepepan del Instituto Politécnico Nacional, al Sistema Nacional de Investigadores del Consejo Nacional de Ciencia y Tecnología.

\section{Referencias}

[1] M. Blázquez y M. F. Peretti, "Modelo para gestionar la sustentabilidad de las organizaciones a través de la rentabilidad, adaptabilidad e imagen". Estudios Gerenciales, vol. 28, no.125, pp. 40-50, 2012.

[2] Organización Internacional del Trabajo (OIT). La promoción de las empresas sostenibles. Suiza: Organización Internacional de Trabajo. 2007.

[3] M. Espino. Sustentabilidad de las empresas. México: Instituto Mexicano de Contadores Públicos. 2009.

[4] F. Almagro. Cuentas ecológicas y desarrollo sustentable. La experiencia en México. México: Instituto Politécnico Nacional. 2009.

[5] M. Porter y M. Kramer. Estrategia y Sociedad. Harvard Business School America Latina, pp. 1-15. Dic 2006.

[6] G. Cervantes, R. Sosa, G. Rodríguez y F. Robles, "Ecología industrial y desarrollo sustentable", Ingeniería, vol. 13, no. 1, pp. 63-70, 2009.

[7] M. J. Déniz y M. M. Verona, "Gestión ambientalmente responsable y valor de mercado de las acciones en situaciones de crisis financieras", Investigación Económica, vol. 71, no. 281, pp. 117148, 2012.

[8] J. D. Margolis y J. P. Walsh, "Misery Loves Companies: Whither Social Initiatives by Business", Harvard Business School Working Paper, vol. 1, no. 58, pp. 1-62, 2001.

[9] M. Orlitzky, F. Schmidt, y S. Rynes, "Corporate Social and Financial Performance: A Meta-analysis", Organization Studies, vol. 24, no. 3, pp. 403-441, 2003.

[10] Forética. Sostenibilidad parafinancieros. RESEARCH Publicación de tendencias y actualidad RSE, Madrid, No. 4. p. 31. Jun 2011. [Online]. Disponible en: http://www.spainsif.es/sites/default/ files/upload/varios/sostenibilidad_ para_financieros.pdf.

[11] M. J. Charlo y I. Moya, "El 
comportamiento financiero de las empresas socialmente responsables", Investigaciones Europeas de Dirección y Economía de la Empresa, vol. 16, no. 2, pp. 15-25, 2010.

[12] J. C. Van. Administración financiera. México: Prentice Hall Hispanoamericana.1993.

[13] C. Walsh. Ratios fundamentales de gestión empresarial: Cómo analizar y controlar los aspectos que marcan el valor de las empresas. Madrid: Pearson Educación. 2001.

[14] J. Gitman y J. Zutter. Principios de administración financiera. México: Pearson. 2012.

[15] R. W. Jhonson y R. W. Melicher. Administración financiera. México: CECSA. 2000. 